\title{
Addition of oxaliplatin to capecitabine-based preoperative chemoradiotherapy for locally advanced rectal cancer: Long-term outcome of a phase II study
}

\author{
JIANHONG PENG $^{1 *}$, JUNZHONG LIN ${ }^{1 *}$, ZHIFAN ZENG $^{2}$, XIAOJUN WU ${ }^{1}$, GONG CHEN $^{1}$, \\ LIREN LI ${ }^{1}$, ZHENHAI LU $^{1}$, PEIRONG DING ${ }^{1}$, DESEN WAN ${ }^{1}$ and ZHIZHONG PAN ${ }^{1}$ \\ Departments of ${ }^{1}$ Colorectal Surgery and ${ }^{2}$ Radiation Oncology, Sun Yat-sen University Cancer Center, \\ State Key Laboratory of Oncology in South China, Collaborative Innovation Center of \\ Cancer Medicine, Guangzhou, Guangdong 510060, P.R. China
}

Received December 28, 2015; Accepted June 2, 2017

DOI: $10.3892 / \mathrm{ol} .2017 .6764$

\begin{abstract}
Our previous study reported the favorable short-term outcome and good tolerance of integrating oxaliplatin into capecitabine-based (XELOX regimen) preoperative chemoradiotherapy (CRT) for locally advanced rectal cancer (LARC). The present study reported the long-term oncological outcome of this phase II study. A total of 47 patients with rectal adenocarcinoma (stage II or III) were enrolled and received radiotherapy (46 Gy in 23 fractions) in combination with capecitabine $\left(1,000 \mathrm{mg} / \mathrm{m}^{2}\right.$, twice daily, on days $1-14$ and 22-35) and oxaliplatin (130 mg/m $\mathrm{m}^{2}$ on days 1 and 22). Overall survival (OS) rate, disease-free survival (DFS) rate and cumulative incidence of recurrences and long-term complications were calculated or observed. As a result, 41 patients underwent surgery after preoperative CRT, and the cumulative OS rates at 1, 3 and 5 years for these patients were 100.0, 84.5 and $81.8 \%$, respectively. For the 38 patients who received R0 resection, the cumulative OS rates at 1, 3 and 5 years were 100.0, 89.0 and $86.2 \%$, respectively, while the cumulative DFS rates at 1 , 3 and 5 years were 94.6, 75.3 and $69.7 \%$, respectively. After follow-up at 84 months, the cumulative incidence rates of local and distant recurrences at 5 years were 6.6 and $28.2 \%$, respectively. Oxaliplatin-associated long-term complications were seldom observed. Overall, the addition of oxaliplatin to capecitabine-based preoperative radiotherapy achieved
\end{abstract}

Correspondence to: Professor Zhizhong Pan, Department of Colorectal Surgery, Sun Yat-sen University Cancer Center, State Key Laboratory of Oncology in South China, Collaborative Innovation Center of Cancer Medicine, 651 East Dongfeng Road, Guangzhou, Guangdong 510060, P.R. China

E-mail: panzhzh@sysucc.org.cn

*Contributed equally

Key words: oxaliplatin, preoperative chemoradiotherapy, rectal cancer, long-term outcome favorable OS and DFS without increased long-term complications in patients with LARC. Therefore, this preoperative CRT strategy is a feasible option for such patients.

\section{Introduction}

To date, 5-fluorouracil (5-FU)-based concurrent chemoradiotherapy (CRT) followed by total mesorectal excision (TME) has been the standard treatment for locally advanced rectal cancer (LARC) (1,2). Although local tumor regression was distinctly observed in the majority of patients following CRT, distant metastases remained the main cause for failure, possibly due to the insufficient control of systemic micro-metastasis by CRT $(3,4)$. To enhance local and systemic control, our previous study applied oxaliplatin into capecitabine-based (XELOX regimen) preoperative CRT, which was demonstrated to be feasible and well tolerated (5). The short-term result of this study demonstrated a favorable pathological complete response (pCR; 22\%, 9/41), which was consistent with previous studies in the same setting (6-8).

Several phase II studies using the XELOX regimen combined with radiotherapy presented $\mathrm{pCR}$ as the primary endpoint $(9,10)$. However, the early surrogate endpoint of pCR may not completely reflect the authentic clinical efficacy of CRT for LARC (11). It is well-known that overall survival (OS) is the determinant endpoint in a clinical study $(12,13)$. Thus, long-term follow-up data are required to draw specific conclusions with respect to the rates of local recurrence and distant metastases. With a median follow-up time of 84 months, the present study investigated the 5-year OS and disease-free survival (DFS) rates, the cumulative incidence of local and distant recurrences, and the long-term complications found in patients with LARC who underwent preoperative chemoradiotherapy with the XELOX regimen in our previous phase II study (5).

\section{Patients and methods}

Patients and methods. The prospective, single arm phase II study (clinical trial number ChiCTR-OIC-17011632) was 


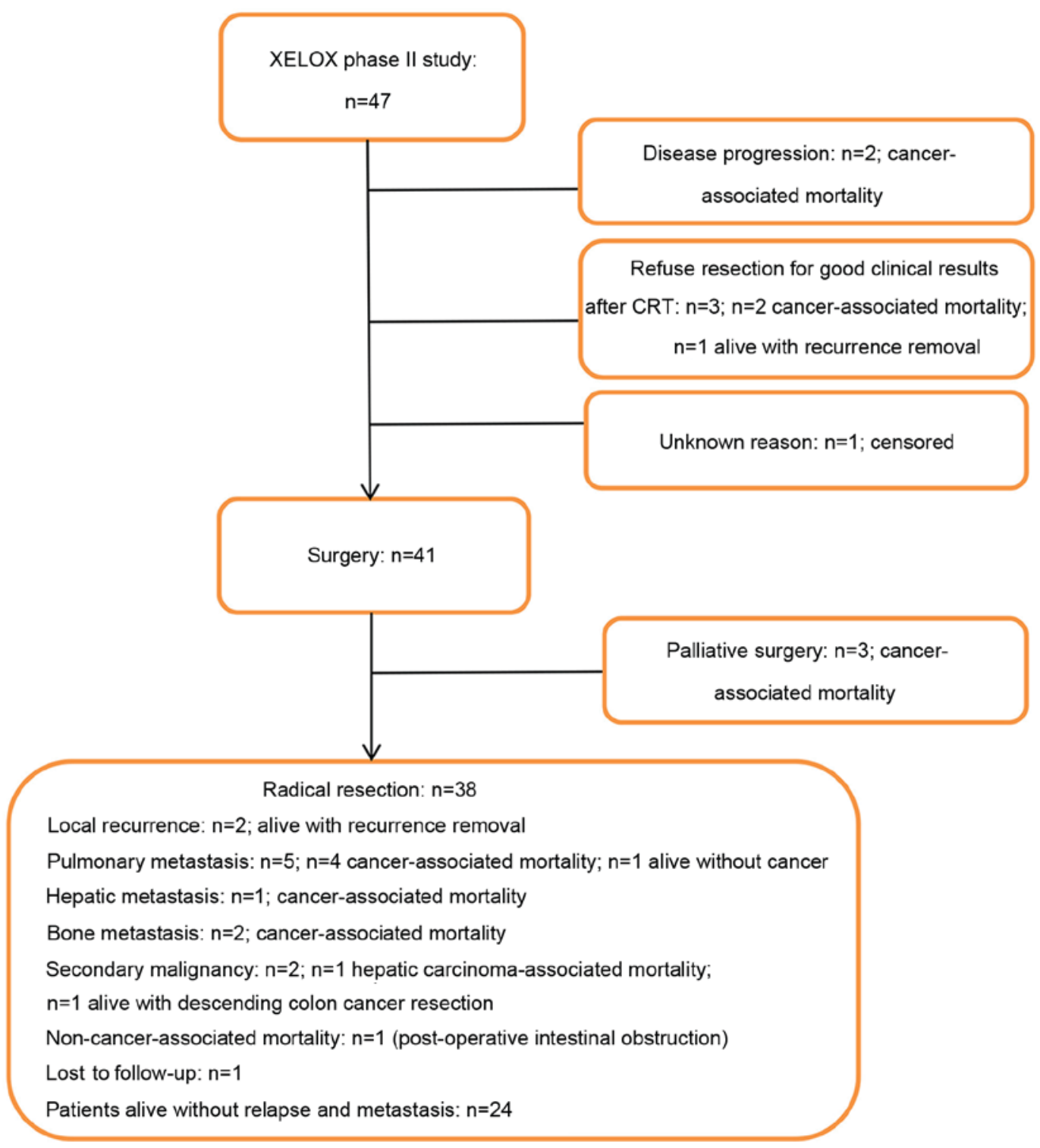

Figure 1. Summary of the XELOX phase II study. Of the total 47 patients, 41 patients received surgery for the primary rectal cancer and 38 patients (92.7\%) were treated with radical resection of the primary tumor. CRT, chemoradiotherapy; XELOX, capecitabine plus oxaliplatin.

conducted between March 2007 and June 2008 at the Cancer Center of Sun Yat-sen University (Guangzhou, China). We have previously reported details of the study, including eligibility criteria, evaluation method, treatment model, pathological analysis and short-term endpoints (pCR rate), CRT-associated toxicities, $\mathrm{R} 0$ resection rates, sphincter-sparing rates and 1-month surgical complications (5). Patients with stage II and III (T3-T4 and/or $\mathrm{N}^{+}$) histologically confirmed rectal adenocarcinomas received radiotherapy (46 Gy in 23 fractions) in combination with capecitabine $\left(1,000 \mathrm{mg} / \mathrm{m}^{2}\right.$, twice daily, on days $1-14$ and 22-35) and oxaliplatin (130 $\mathrm{mg} / \mathrm{m}^{2}$ on days 1 and 22$)$. TME surgery was scheduled to take place 4-6 weeks after completion of preoperative CRT. Either six cycles of XELOX regimen or four cycles of XELOX regimen plus two cycles of capecitabine, was recommended for patients 4 weeks after surgery. All patients provided written informed consent and the Ethical Committee of Sun Yat-sen University Cancer Center approved the study protocol. The study was performed in accordance with the ethical standards of the World Medical Association Declaration of Helsinki.

Follow-up. All patients were observed through subsequent visits every 3 months for 2 years, and then semi-annually until 5 years post-surgery. Evaluation included clinical examination, carcinoembryonic antigen level, abdominal ultrasonography and chest radiography. Chest computed tomography, abdom$\mathrm{inal} /$ pelvic magnetic resonance imaging and colonoscopy were performed annually. Recurrence in the pelvis was defined as local recurrence, and recurrence outside the pelvis was considered as distant metastasis.

Study endpoints. The primary endpoints of the XELOX phase II study, including tumor regression and toxicities, were reported previously (5). Long-term secondary endpoints included OS, DFS and the cumulative incidence of recurrences. OS time was calculated from the beginning of surgery to rectal cancer-associated mortality or the time of 
last follow-up. DFS time was measured between the date of radical surgery and the date of diagnosis of local recurrence and distant metastasis from rectal cancer. Local and distant recurrence analyses were performed on all eligible patients who underwent a complete local resection (patients only with $\mathrm{R} 0$ resection of the primary tumor were included, whereas patients with $\mathrm{R} 1$ and $\mathrm{R} 2$ resection of the primary tumor were excluded). Long-term complication was defined according to the literature as occurring or persisting 6 months after surgery for primary rectal cancer (14).

Statistical analysis. Statistical analysis was performed with the SPSS ${ }^{\circledR}$ statistical package for Windows (version 17.0; SPSS, Inc., Chicago, IL, USA). Continuous variables are summarized as the median (range) and categorical variables are presented as percentages. Analyses for recurrences were reported as cumulative incidence rates. Kaplan-Meier methodology was applied to calculate OS, DFS and cumulative incidence rates by performing survival curves. All tests were two-tailed, in which $\mathrm{P}<0.05$ was considered to indicate a statistically significant difference.

\section{Results}

Patient characteristics. In total, 47 patients were enrolled in the present study and received preoperative CRT as planned. As shown in Fig. 1, 6 patients did not undergo surgery; 2 patients were diagnosed with an unresectable tumor with hepatic metastases following preoperative CRT and then administered palliative chemotherapy, 3 patients refused to receive surgery due to good tumor regression after CRT and 1 patient did not undergo surgery for an unknown reason. As a result, 41 patients $(87.2 \%)$ received surgery following preoperative CRT. The clinical baseline characteristics of those patients are shown in Table I. Primary tumor palliative resection was performed in 3 patients: 1 patient was diagnosed with hepatic metastasis following CRT, and pelvic metastases were detected intraoperatively in the other 2 patients. In total, radical resection was performed on 38 patients $(92.7 \%)$. Clinicopathological parameters subsequent to treatments are presented in Table II. T stage downstaging was observed in 24 out of 41 patients $(58.5 \%)$ and Union for International Cancer Control downstaging was observed in 25 of 41 patients (61.0\%) (15). A pCR was achieved in 9 out of 41 patients (22.0\%) (5). In total, 33 patients $(80.5 \%)$ subsequently received post-surgery adjuvant chemotherapy, with a median of 6 cycles (1-6 cycles), $60.6 \%(20 / 33)$ of whom underwent complete adjuvant chemotherapy as planned.

Long-term postoperative complications. A total of 41 patients who underwent surgery following preoperative CRT were evaluated for long-term postoperative complications. Long-term complications were observed in 14 of 41 patients (34.1\%). As shown in Table III, 4 patients (9.8\%) experienced sexual dysfunction, 3 patients $(7.3 \%)$ suffered a severe defecation disorder, 3 patients $(7.3 \%)$ had sequential acroanesthesia due to peripheral nerve toxicity for 5 years, 2 patients $(4.9 \%)$ were found to have anastomotic stenosis through colonoscopy following 6 months of postoperative conservative treatment,

Table I. Baseline characteristics of patients undergoing surgery.

Characteristics

Median age (range), years

Sex, n $(\%)$

Male

Female

Tumor distance from anal verge, $\mathrm{n}(\%)$

$<6 \mathrm{~cm}$

$6-10 \mathrm{~cm}$

Median primary tumor size (range), $\mathrm{cm}$

Clinical TNM stage, n (\%)

II

III

cT stage, n (\%)

T3

$\mathrm{T} 4$

cN stage, n (\%)

NO

N1

N2
Patient values $(n=41)$

53 (26-75)

$26(63.4)$

15 (36.6)

27 (65.9)

14 (34.1)

$4.5(2-8)$

13 (31.7)

$28(68.3)$

25 (61.0)

16 (39.0)

13 (31.7)

16 (39.0)

12 (29.3)

TNM, tumor-node-metastasis; cT stage, clinical tumor stage; $\mathrm{cN}$ stage, clinical node stage.

2 patients $(4.9 \%)$ were diagnosed with hepatic carcinoma and descending colon cancer after treatment, and 1 patient $(2.4 \%)$ succumbed to reiterant intestinal obstruction with uropoiesis dysfunction.

Recurrence and survival parameters. During the median 84-month follow-up period (range, 1-99 months), the survival outcome of the 6 patients who failed to receive surgery was as followed: 1 patient was lost follow-up, 2 patients succumbed at 1 and 13 months after CRT due to disease progression, and of the 3 patients who refused surgery due to a favorable CRT results, 2 succumbed at 8 and 14 months, respectively, due to disease progression, and the other patient who had local recurrence and then underwent lesion removal survived. Of those patients who underwent surgery $(n=41)$, the 3 patients who underwent $\mathrm{R} 2$ resection succumbed to tumor progression after 6, 15 and 17 months, respectively. Ultimately, 38 patients who underwent R0 were evaluated for recurrences (Fig. 1). In this cohort, 2 patients $(5.3 \%)$ developed local recurrences, with a $6.6 \%$ cumulative incidence rate of local recurrences at 5 years (Fig. 2A). The median local recurrence time was 25 months (range, 18-32 months). The cumulative incidence rate of distant recurrence at 5 years was $28.2 \%$ (Fig. 2B). The median distant recurrence time was 16 months (range, 2-57 months). In total, $8(21.1 \%)$ patients developed distant metastases, including pulmonary metastases (13.2\%), hepatic metastases (2.6\%) and bone metastases $(5.3 \%)$. For patients receiving surgery $(n=41)$, OS rates at 1, 3 and 5 years were $100.0,84.5$ and $81.8 \%$, respectively (Fig. 3A). In the patients who received an $\mathrm{R} 0$ resection $(n=38)$, OS rates at 1,3 and 5 years were 100.0, 89.0, 86.2\%, 
Table II. Clinicopathological parameters after preoperative chemoradiotherapy followed by surgery.

\begin{tabular}{lc} 
Patients parameters & Patient values (n \\
\hline Type of surgery, $\mathrm{n}(\%)$ & \\
Anterior resection & $21(51.2)$ \\
Abdominal perineal resection & $17(41.5)$ \\
Hartmann's operation & $2(4.9)$ \\
Resection of adjacent organs ${ }^{\mathrm{a}}, \mathrm{n}(\%)$ & $1(2.4)$ \\
Resection status, $\mathrm{n}(\%)$ & \\
R0 & $38(92.7)$ \\
R1 & $0(0.0)$ \\
R2 & $3(7.3)$ \\
Median number of investigated & $7(1-27)$ \\
lymph nodes (range) & \\
Pathological ypTNM stages, $\mathrm{n}(\%)$ & \\
ypT0N0M0 & $9(22.0)$ \\
ypT1-2N0M0 & $7(17.1)$ \\
ypT3-4N0M0 & $12(29.3)$ \\
ypT1-4N1-2M0 & $10(24.4)$ \\
ypTxNxM1 & $3(7.3)$ \\
Tumor regression grading, $\mathrm{n}(\%)$ & \\
4 (complete regression) & $9(22.0)$ \\
3 (>50\% of tumor mass) & $10(24.4)$ \\
2 (25-50\% of tumor mass) & $10(24.4)$ \\
1 (<25\% of tumor mass) & $9(22.0)$ \\
0 (no regression) & $3(7.3)$ \\
Post-operative chemotherapy & \\
None & $8(19.5)$ \\
XELOX regimen & $15(36.6)$ \\
XELOX + capecitabine & $14(34.1)$ \\
Capecitabine regimen & $3(7.3)$ \\
FOLFOX regimen & $1(2.4)$
\end{tabular}

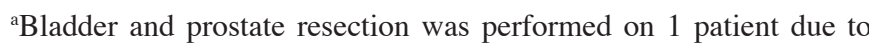
tumor invasion. ypTNM stage, pathological tumor-node-metastasis stage following chemoradiotherapy; FOLFOX, folinic acid, fluorouracil and oxaliplatin; XELOX, capecitabine plus oxaliplatin.

respectively (Fig. 3B), and DFS rates at 1, 3 and 5 years were 94.6, 75.3, 69.7\%, respectively (Fig. 3C).

\section{Discussion}

The optimal chemotherapeutic selection for preoperative CRT in LARC remains an ongoing issue (16). Although preoperative CRT with 5-FU-based regimen has substantially reduced the risk of local recurrence, systemic failure remains the major challenge in the management of LARC $(17,18)$. Previous clinical trials have focused on intensification of conventional chemotherapy by the addition of a second cytotoxic drug to a fluorouracil backbone $(19,20)$. As a potent radiosensitizer, oxaliplatin has been experimentally shown to enhance cytotoxicity and radiosensization for treating rectal cancer,
Table III. Long-term complications after preoperative chemoradiotherapy followed by surgery in patients with local advanced rectal cancer.

Complications Patient values, n (\%)

Defecation disorder

$3(7.3)$

Uropoiesis dysfunction

$1(2.4)$

Anastomotic stenosis

2 (4.9)

Postoperative obstruction

$1(2.4)$

Femoral head necrosis

$0(0.0)$

Sexual dysfunction

4 (9.8)

Second primary malignancy ${ }^{\mathrm{a}}$

2 (4.9)

Peripheral nerve toxicity

$3(7.3)$

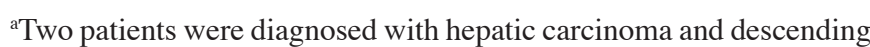
colon cancer after preoperative chemoradiotherapy followed by surgery, respectively.

which have become important components in preoperative chemotherapy regimens $(21,22)$. In order to improve clearance of the primary tumor and systemic micro-metastasis, and to ultimately translate this into a survival improvement, oxaliplatin was added to capecitabine-based preoperative CRT, and short-term and long-term efficacies were then evaluated for this strategy. The final results demonstrated that integration of oxaliplatin and capecitabine to CRT followed by TME surgery was feasible, with good compliance, acceptable toxicity and low surgical morbidity (5). With a median follow-up time of 84 months, favorable 5-year DFS (69.7\%) and OS (86.2\%) rates were obtained from the present study, which was consistent with previous studies of oxaliplatin-based preoperative CRT for treating $\operatorname{LARC}(23,24)$.

It has been shown that preoperative CRT with the XELOX regimen, followed by optimized TME surgery, markedly achieved a higher pCR rate and decreased local recurrence rate of $5-8 \%$, whereas the distant metastatic rate in LARC following TME surgery was 3-6 times higher than the local recurrent rate in previous clinical trials and in the present study $(25,26)$. However, the value of controlling distant metastasis by performing oxaliplatin-based preoperative CRT remains uncertain. The $\mathrm{CAO} / \mathrm{ARO} / \mathrm{AIO}-04$ trial showed that the cumulative incidence of distant recurrences at 3 years after R0/1 resection was $18.5 \%$ [95\% confidence interval (CI), 15.2-21.7] in an oxaliplatin group and $22.4 \%$ (95\% CI, 19.1-25.8) in a non-oxaliplatin group. Although a numerical difference could be observed, it failed to reach statistical significance (27). The STAR-01 trial reported that integrating oxaliplatin into a capecitabine-based preoperative CRT regimen could contribute to a reduced percentage of intra-abdominal metastases $(0.5 \%$ with oxaliplatin vs. $2.9 \%$ without; $\mathrm{P}=0.014$ ) (28). However, the incidence of pulmonary metastasis in the CAPEOX group in the ACCORD 12/0405 PRODIGE 2 trial was not less than that of the capecitabine group at 3 years (11.0 vs. 10.4\%) (29). Similar to the uncertain impact of oxaliplatin in distant metastasis, the benefit of adding oxaliplatin to preoperative CRT for a significant improvement in long-term survival has also not been specifically confirmed 


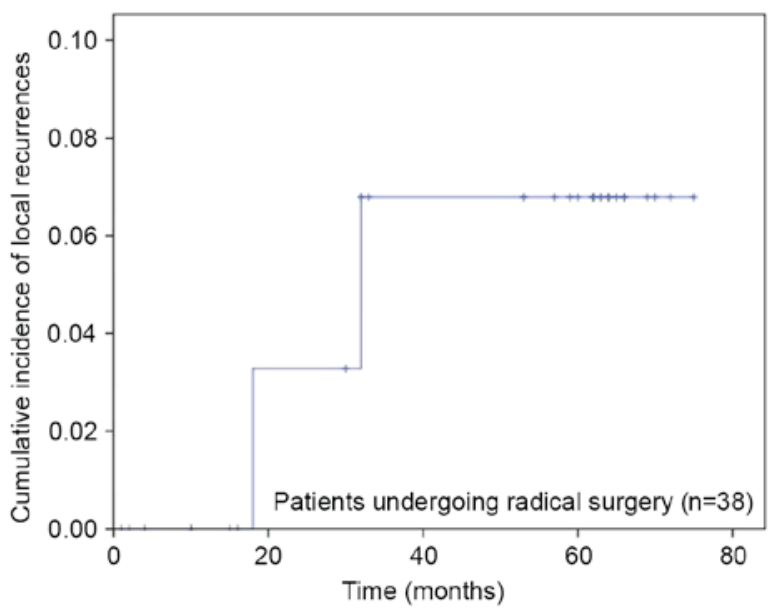

B

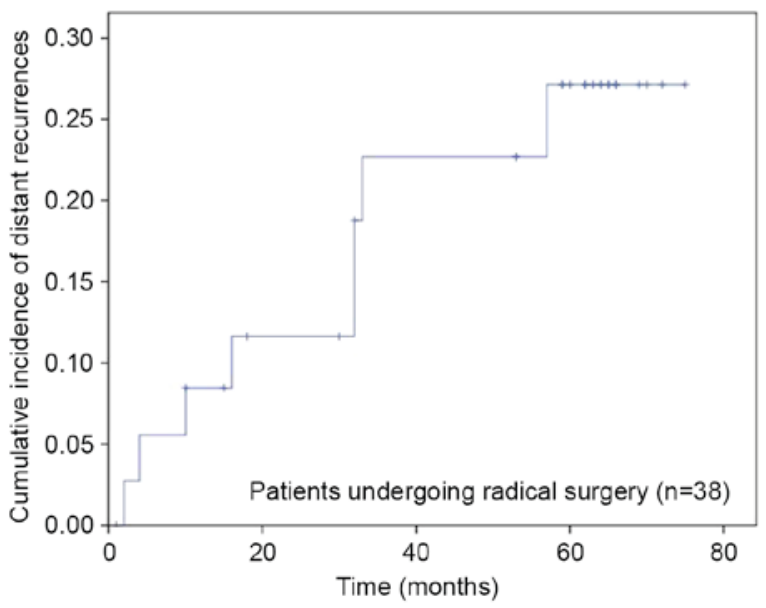

Figure 2. Kaplan-Meier curves showing the (A) cumulative incidence of local recurrences and (B) the cumulative incidence of distant recurrences of patients with locally advanced rectal cancer treated by preoperative chemoradiotherapy plus radical resection in the present study.

thus far. As shown in Table IV, the addition of oxaliplatin delivered different 3-year DFS rates (range, 60.0-78.6\%) and 5 -year OS rates (range, 54.7-92.0\%) (23-27,29-31). Among those phase III studies, only the CAO/ARO/AIO-04 trial demonstrated a survival benefit from adding oxaliplatin to preoperative treatment (DFS at 3 years: $75.9 \%$ with oxaliplatin vs. $71.2 \%$ without; $\mathrm{P}=0.03$; hazard ratio, $0.79 ; 95 \% \mathrm{CI}$, 0.64-0.98) (27). By contrast, the long-term outcomes of the ACCORD 12/0405-Prodige 2 trial and the NSABP R-04 trial did not reach a statistically significant difference in terms of the 3- and 5-year DFS rates (29,31). All current studies failed to achieve a significant benefit to overall survival by adding oxaliplatin to preoperative CRT $(27,29-31)$. However, it must be noted that the CAO/ARO/AIO-04 trial administered oxaliplatin in preoperative and post-operative chemotherapy, while the ACCORD12/0405-Prodige 2 trial only administered oxaliplatin during preoperative treatment. Furthermore, with the exception of the CAO/ARO/AIO-04 trial, adding oxaliplatin to the conventional CRT led to increased toxicity and reduced tolerance, resulting in a lower dose of oxaliplatin in the combination arm, which ultimately compromised the local and systematic effect of CRT. Therefore, optimizing the
A

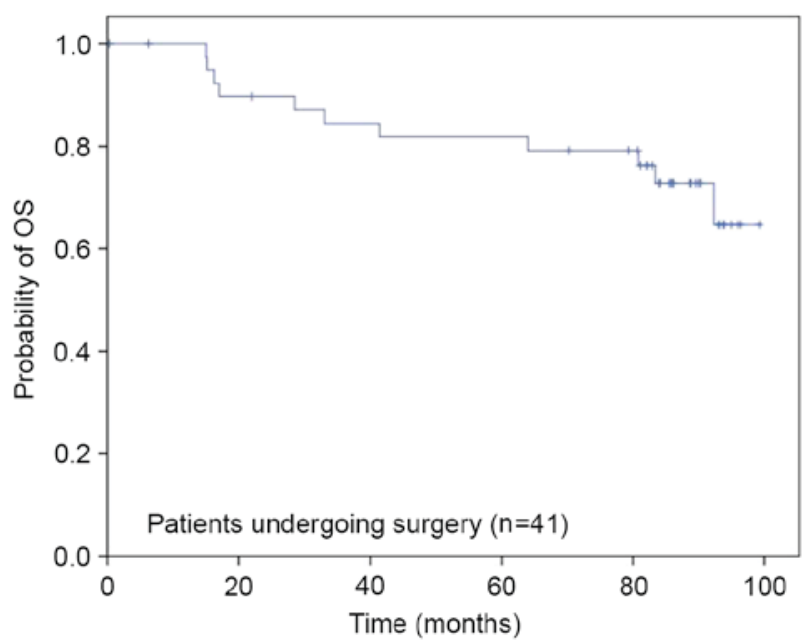

B

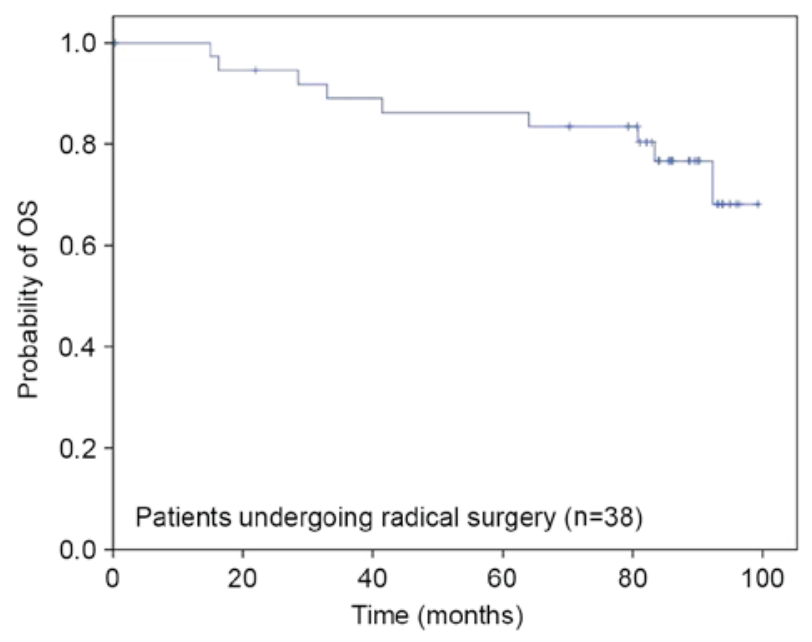

C

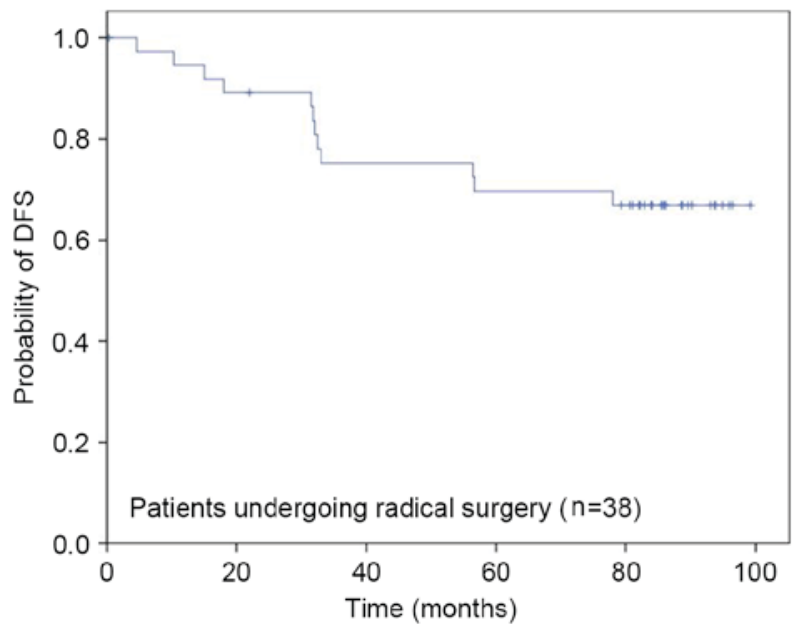

Figure 3. Clinical long-term outcome in patients treated with capecitabine plus oxaliplatin in preoperative chemotherapy. (A) OS in the patients undergoing primary tumor resection (R0 and R2); (B) OS and (C) DFS for patients treated with radical excision surgery following completion of preoperative chemoradiotherapy. OS, overall survival; DFS, disease-free survival.

dose and schedule for administration of oxaliplatin possibly contributes to good tolerance, which may finally result in a favorable long-term outcome. 


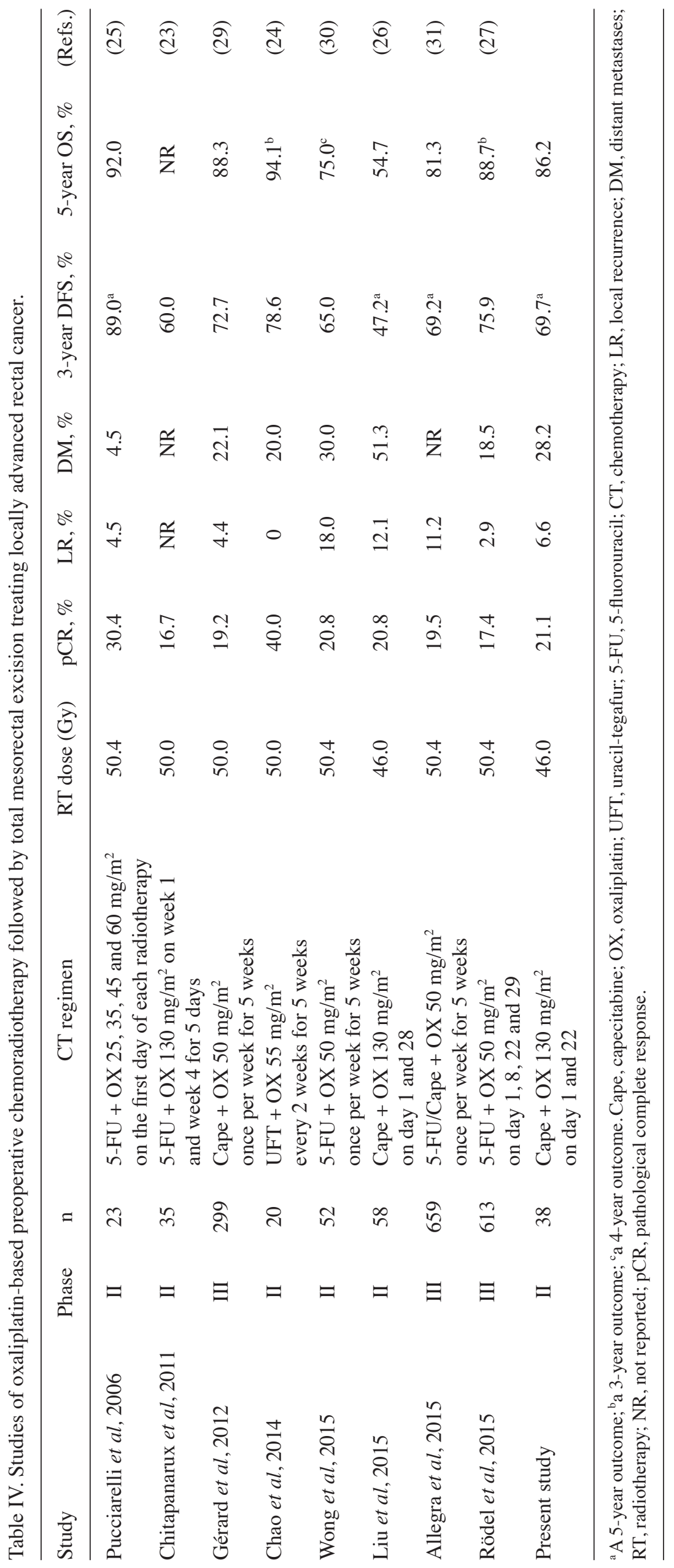


A concern of adding oxaliplatin concomitantly to conventional CRT is that it may significantly increase toxicity, with $\sim 50 \%$ of patients ending up not receiving the complete chemotherapy $(6,7)$ and $13-16 \%$ of patients not receiving the complete dose of radiation $(28,32)$. The current strategy was well tolerated and all patients received the preoperative CRT as planned. The low toxicities and high compliance to treatment may be attributed to the strategy of drug delivery (as aforementioned). In the present study, the XELOX regimen was delivered with a 1-week chemotherapy intermission, which possibly contributed to the good tolerance. In addition, unlike the poor capecitabine tolerability found in the American population $(33,34)$, acceptable toxicities and compliance were found in the Chinese patients in the current study, when administered the standard dose of capecitabine $\left(1,000 \mathrm{mg} / \mathrm{m}^{2}\right.$, twice daily, on days $1-14$ of a 3-week schedule). With a median follow-up time of 84 months, the major long-term complications, including sexual dysfunction (9.8\%), defecation disorders (7.3\%) and peripheral nerve toxicity (7.3\%), were observed without lethal cause in the present study. The long-term surgical complications were comparable to those in other studies using conventional 5-FU-based CRT $(1,4)$. With the exception of peripheral nerve toxicity, it was considered that long-term complications may be to a great extent relevant to the postoperatively shortened intestinal length, diminished rectal reservoir and damage to the sphincter complex or its innervation, but not the toxicity of oxaliplatin.

There were several limitations in the present study, including the small number of patients and selection bias. Consequently, randomized control trials are required to determine the definite role of the current strategy. In addition, $19.5 \%$ of the patients in the present study failed to receive post-operative chemotherapy, which may impact the long-term outcome to a large extent (35). In addition, despite the satisfactory median follow-up period of the present study (84 months), the follow-up time is insufficient to measure the 10-year long-term outcome for patients with LARC. Longer outcome data are required for this setting.

In conclusion, addition of oxaliplatin into capecitabine-based preoperative radiotherapy could achieve favorable OS and DFS rates without increasing long-term oxaliplatin-associated complications in LARC. Distant recurrence remains the predominant pattern of failure after preoperative CRT followed by TME.

\section{Acknowledgements}

This study was supported by grants from the Sun Yat-sen University Clinical Research 5010 Program (no. 2015024) and a grant of Guangzhou Science and Technology Plan Projects (Health Medical Collaborative Innovation Program of Guangzhou) (grant no. 201400000001-4).

\section{References}

1. Bosset JF, Collette L, Calais G, Mineur L, Maingon P, Radosevic-Jelic L, Daban A, Bardet E, Beny A and Ollier JC; EORTC Radiotherapy Group Trial 22921: Chemotherapy with preoperative radiotherapy in rectal cancer. N Engl J Med 355: 1114-1123, 2006.

2. Roh MS, Colangelo LH, O'Connell MJ, Yothers G, Deutsch M, Allegra CJ, Kahlenberg MS, Baez-Diaz L, Ursiny CS, Petrelli NJ and Wolmark N: Preoperative multimodality therapy improves disease-free survival in patients with carcinoma of the rectum: NSABP R-03. J Clin Oncol 27: 5124-5130, 2009.
3. Sanfilippo NJ, Crane CH, Skibber J, Feig B, Abbruzzese JL, Curley S, Vauthey JN, Ellis LM, Hoff P, Wolff RA, et al: T4 rectal cancer treated with preoperative chemoradiation to the posterior pelvis followed by multivisceral resection: Patterns of failure and limitations of treatment. Int J Radiat Oncol Biol Phys 51: 176-183, 2001.

4. Sauer R, Becker H, Hohenberger W, Rödel C, Wittekind C, Fietkau R, Martus P, Tschmelitsch J, Hager E, Hess CF, et al: Preoperative versus postoperative chemoradiotherapy for rectal cancer. N Engl J Med 351: 1731-1740, 2004.

5. Lin JZ, Zeng ZF, Wu XJ, Wan DS, Chen G, Li LR, Lu ZH, Ding PR and Pan ZZ: Phase II study of pre-operative radiotherapy with capecitabine and oxaliplatin for rectal cancer and carcinoembryonic antigen as a predictor of pathological tumour response. J Int Med Res 38: 645-654, 2010.

6. Zhao L, Bai C, Shao Y, Guan M, Jia N, Xiao Y, Qiu H, Zhang F, Yang T, Zhong G and Chen S: A phase II study of neoadjuvant chemoradiotherapy with oxaliplatin and capecitabine for rectal cancer. Cancer Lett 310: 134-139, 2011.

7. Koeberle D, Burkhard R, von Moos R, Winterhalder R, Hess V, Heitzmann F, Ruhstaller T, Terraciano L, Neuweiler J, Bieri G, et al: Phase II study of capecitabine and oxaliplatin given prior to and concurrently with preoperative pelvic radiotherapy in patients with locally advanced rectal cancer. Br J Cancer 98: 1204-1209, 2008.

8. Aschele C, Friso ML, Pucciarelli S, Lonardi S, Sartor L, Fabris G, Urso ED, Del Bianco P, Sotti G, Lise M and Monfardini S: A phase I-II study of weekly oxaliplatin, 5-fluorouracil continuous infusion and preoperative radiotherapy in locally advanced rectal cancer. Ann Oncol 16: 1140-1146, 2005.

9. Carlomagno C, Farella A, Bucci L, D'Armiento FP, Pesce G, Pepe S, Cannella L, Pacelli R, De Stefano A, Solla R, et al: Neo-adjuvant treatment of rectal cancer with capecitabine and oxaliplatin in combination with radiotherapy: A phase II study. Ann Oncol 20: 906-912, 2009.

10. Salazar R, Navarro M, Losa F, Alonso V, Gallén M, Rivera F, Benavides M, Escudero P, González E, Massutí B, et al: Phase II study of preoperative radiotherapy and concomitant weekly intravenous oxaliplatin combined with oral capecitabine for stages II-III rectal cancer. Clin Transl Oncol 14: 592-598, 2012.

11. Bonnetain F, Bosset JF, Gerard JP, Calais G, Conroy T, Mineur L, Bouché O, Maingon P, Chapet O, Radosevic-Jelic L, et al: What is the clinical benefit of preoperative chemoradiotherapy with 5FU/leucovorin for T3-4 rectal cancer in a pooled analysis of EORTC 22921 and FFCD 9203 trials: Surrogacy in question? Eur J Cancer 48: 1781-1790, 2012.

12. Foster NR, Qi Y, Shi Q, Krook JE, Kugler JW, Jett JR, Molina JR, Schild SE, Adjei AA and Mandrekar SJ: Tumor response and progression-free survival as potential surrogate endpoints for overall survival in extensive stage small-cell lung cancer: Findings on the basis of North Central Cancer Treatment Group trials. Cancer 117: 1262-1271, 2011.

13. Oba K, Paoletti X, Alberts S, Bang YJ, Benedetti J, Bleiberg H, Catalano P, Lordick F, Michiels S, Morita S, et al: Disease-free survival as a surrogate for overall survival in adjuvant trials of gastric cancer: A meta-analysis. J Natl Cancer Inst 105: 1600-1607, 2013.

14. Birgisson H, Påhlman L, Gunnarsson U and Glimelius B; Swedish Rectal Cancer Trial Group: Adverse effects of preoperative radiation therapy for rectal cancer: Long-term follow-up of the Swedish Rectal Cancer Trial. J Clin Oncol 23: 8697-8705, 2005.

15. Sobin LH: TNM, sixth edition: New developments in general concepts and rules. Semin Surg Oncol 21: 19-22, 2003.

16. Mohiuddin M, Mohiuddin MM, Marks J and Marks G: Future directions in neoadjuvant therapy of rectal cancer: Maximizing pathological complete response rates. Cancer Treat Rev 35: 547-552, 2009.

17. Gérard JP, Conroy T, Bonnetain F, Bouché O, Chapet O, Closon-Dejardin MT, Untereiner M, Leduc B, Francois E, Maurel J, et al: Preoperative radiotherapy with or without concurrent fluorouracil and leucovorin in T3-4 rectal cancers: Results of FFCD 9203. J Clin Oncol 24: 4620-4625, 2006.

18. Sauer R, Liersch T, Merkel S, Fietkau R, Hohenberger W, Hess C, Becker H, Raab HR, Villanueva MT, Witzigmann H, et al: Preoperative versus postoperative chemoradiotherapy for locally advanced rectal cancer: Results of the German CAO/ARO/AIO-94 randomized phase III trial after a median follow-up of 11 years. J Clin Oncol 30: 1926-1933, 2012. 
19. Glynne-Jones R, Falk S, Maughan TS, Meadows HM and Sebag-Montefiore D: A phase I/II study of irinotecan when added to 5-fluorouracil and leucovorin and pelvic radiation in locally advanced rectal cancer: A Colorectal Clinical Oncology Group Study. Br J Cancer 96: 551-558, 2007.

20. Gao YH, An X, Sun WJ, Cai J, Cai MY, Kong LH, Lin JZ, Liu GC, Tang JH, Wu XJ, et al: Evaluation of capecitabine and oxaliplatin administered prior to and then concomitant to radiotherapy in high risk locally advanced rectal cancer. J Surg Oncol 109: 478-482, 2014.

21. Hermann RM, Rave-Fränk M and Pradier O: Combining radiation with oxaliplatin: A review of experimental results. Cancer Radiother 12: 61-67, 2008.

22. Martin LK and Bekaii-Saab T: Optimizing neoadjuvant therapy for rectal cancer with oxaliplatin. J Natl Compr Canc Netw 11: 298-307, 2013.

23. Chitapanarux I, Chitapanarux T, Tharavichitkul E, Mayurasakorn S, Siriwittayakorn P, Yamada S and Lorvidhaya V: A phase II study of oxaliplatin with 5-FU/folinic acid and concomitant radiotherapy as a preoperative treatment in patients with locally advanced rectal cancer. Biomed Imaging Interv J 7 : e25, 2011. doi: 10.2349/biij.7.4.e25.

24. Chao JY, Wang HM, Chiang FF, Lin JC, Chang CF, Lin JF and Yeh HL: Preoperative chemoradiotherapy with oxaliplatin and tegafur-uracil in locally advanced rectal cancer: Pathologic complete response rate and preliminary results of overall and disease-free survival in a single institute in Taiwan. J Chin Med Assoc 77: 128-132, 2014.

25. Pucciarelli S, Urso E, DeSalvo GL, Aschele C, Friso ML, Rugge M, Toppan P, Bruttocao A, Fabris G, Ferraro B, et al 5-fluorouracil and weekly oxaliplatin combined with radiotherapy for locally advanced rectal cancer: Surgical complications and long-term results. Arch Med Res 37: 860-865, 2006

26. Liu L, Cao C, Zhu Y, Li D, Feng H, Luo J, Tang Z, Liu P, Lu K, $\mathrm{Ju} \mathrm{H}$ and Zhang N: Preoperative chemoradiotherapy with 5-fluorouracil and oxaliplatin for locally advanced rectal cancer: Long-term results of a phase II trial. Med Oncol 32: 70, 2015.

27. Rödel C, Graeven U, Fietkau R, Hohenberger W, Hothorn T, Arnold D, Hofheinz RD, Ghadimi M, Wolff HA Lang-Welzenbach M, et al: Oxaliplatin added to fluorouracil-based preoperative chemoradiotherapy and postoperative chemotherapy of locally advanced rectal cancer (the German CAO/ARO/AIO-04 study): Final results of the multicentre, open-label, randomised, phase 3 trial. Lancet Oncol 16: 979-989, 2015.
28. Aschele C, Cionini L, Lonardi S, Pinto C, Cordio S, Rosati G, Artale S, Tagliagambe A, Ambrosini G, Rosetti P, et al: Primary tumor response to preoperative chemoradiation with or without oxaliplatin in locally advanced rectal cancer: Pathologic results of the STAR-01 randomized phase III trial. J Clin Oncol 29: 2773-2780, 2011.

29. Gérard JP, Azria D, Gourgou-Bourgade S, Martel-Lafay I, Hennequin C, Etienne PL, Vendrely V, Francois E, de La Roche G, Bouché O, et al: Clinical outcome of the ACCORD 12/0405 PRODIGE 2 randomized trial in rectal cancer. J Clin Oncol 30: 4558-4565, 2012.

30. Wong SJ, Moughan J, Meropol NJ, Anne PR, Kachnic LA, Rashid A, Watson JC, Mitchell EP, Pollock J, Lee RJ, et al: Efficacy endpoints of radiation therapy group protocol 0247: A randomized, phase 2 study of neoadjuvant radiation therapy plus concurrent capecitabine and irinotecan or capecitabine and oxaliplatin for patients with locally advanced rectal cancer. Int J Radiat Oncol Biol Phys 91: 116-123, 2015.

31. Allegra CJ, Yothers G, O'Connell MJ, Beart RW, Wozniak TF, Pitot HC, Shields AF, Landry JC, Ryan DP, Arora A, et al: Neoadjuvant 5-FU or capecitabine plus radiation with or without oxaliplatin in rectal cancer patients: A phase III randomized clinical trial. J Natl Cancer Inst 107: djv248, 2015. doi: 10.1093/jnci/djv248.

32. Gérard JP, Azria D, Gourgou-Bourgade S, Martel-Laffay I, Hennequin C, Etienne PL, Vendrely V, François E, de La Roche G, Bouché O, et al: Comparison of two neoadjuvant chemoradiotherapy regimens for locally advanced rectal cancer: Results of the phase III trial ACCORD 12/0405-Prodige 2. J Clin Oncol 28: 1638-1644, 2010.

33. Grothey A: A comparison of XELOX with FOLFOX-4 as first-line treatment for metastatic colorectal cancer. Nat Clin Pract Oncol 6: 10-11, 2009.

34. Hochster HS, Hart LL, Ramanathan RK, Childs BH, Hainsworth JD, Cohn AL, Wong L, Fehrenbacher L, Abubakr Y, Saif MW, et al: Safety and efficacy of oxaliplatin and fluoropyrimidine regimens with or without bevacizumab as first-line treatment of metastatic colorectal cancer: Results of the TREE study. J Clin Oncol 26: 3523-3529, 2008.

35. Hong YS, Nam BH, Kim KP, Kim JE, Park SJ, Park YS, Park JO, Kim SY, Kim TY, Kim JH, et al: Oxaliplatin, fluorouracil, and leucovorin versus fluorouracil and leucovorin as adjuvant chemotherapy for locally advanced rectal cancer after preoperative chemoradiotherapy (ADORE): An open-label, multicentre, phase 2, randomised controlled trial. Lancet Oncol 15: 1245-1253, 2014. 